\section{Standardisation in human cytogenetics}

AT the Fourth International Conference on Standardisation in Human Genetics, held in Paris in 1971, a Standing Committee was appointed to examine problems relating to standardisation in human cytogenetics and to make recommendations thereon between international standardisation conferences.

During the past four years the Standing Committee has been responsible for publication of the Report of the Paris Conference and a supplement to that report. In addition, the Standing Committee sponsored a workshop on human cytogenetic registries, held in Edinburgh in April 1975 (Nature, 256, 456; 1975) and on the recommendation of this workshop, appointed an international advisory committee on cytogenetic registries

The terms of reference of the Standing Committee call for elections to a new committee no later than the International Congress of Human Genetics in October, 1976 and the possibility of holding the next standardisation conference at that time. For these reasons the committee has recently circulated recommendations to the participants of the Paris Conference, which have been approved as follows: (1) That a standardisation conference on the lines of the Paris and Chicago Conferences could not be justified in 1976 and, therefore, that no such confer- ence should be held in conjunction with the Fifth International Congress of Human Genetics in Mexico City in October, 1976; (2) That a Standing Committee charged with the task of monitoring developments relating to standardisation and nomenclature in human cytogenetics should be maintained; (3) That an open meeting of all interested human cytogeneticists should be held at the Fifth International Congress of Human Genetics in Mexico City in October, 1976. At this meeting a report of the Standing Committee would be received and a new Standing Committee elected. The existing Standing Committee will be acting as a nominating committee and will be preparing a slate of nominations for consideration by the meeting in Mexico City. These nominations will take into account regional and sectional interests to ensure, so far as possible, a fully representative Standing Committee.

Suggestions for nominations should be sent to the Standing Committee. The time and place of the Mexico City meeting will be announced later and circulated to those requesting this information. Nominations and requests for further information should be addressed to: Dr John L. Hamerton, Department of Genetics, Health Sciences Children's Centre, 685 Bannatyne Avenue, Winnipeg, Manitoba, R3E OW1 Canada. effect is still prevalent, since roughly 999 out of 1,000 dopant atoms still seem to be suppressed. It remains to be seen whether this apparent inefficiency in doping can be improved on. The important discovery, however, is the fact that even a few dopant atoms are not suppressed. Junction action is thereby produced and a new device technology may even be in the making. The extent to which this technology could compete with singlecrystal semiconductor technology depends less on the inefficiency of doping mentioned above, which can be countered, than on the achievement of low minority-carrier recombination-generation rates in the junction regions. High rates lead to high reverse currents in rectifiers and loss of efficiency in photovoltaic applications. It will be a much more challenging task to make these recombination rates suitably low but success in doing so could have a greater impact than the muchresearched "amorphous threshold switch' technology, which now seems to have lost any commercial momentum it ever had. An amorphous $p-n$ junction technology would have much in common with the increasingly popular silicon-on-sapphire technology except that the serious restrictions in area imposed by the dimensions of sapphire crystals would have been removed and the way thus opened for integrated circuits as large as the page on which this column is written.

\section{Superfluid within superfluid}

\section{from P. V. E. McClintock}

AT last, somebody has gone out on a limb and named an encouraging number for the temperature of the anticipated superfluid transition among the ${ }^{3} \mathrm{He}$ atoms of a liquid ${ }^{3} \mathrm{He}-{ }^{4} \mathrm{He}$ solution. According to Patton and Zaringhalam of the Massachusetts Institute of Technology, writing in a recent issue of Physics Letters $(\mathbf{5 5} \mathbf{A}$, $95 ;$ 1975), the transition is likely to take place under conditions which can be achieved experimentally.

They are brave men. The first estimates of the superfluid transition temperature $T_{\mathrm{c}}$ of pure liquid ${ }^{3} \mathrm{He}$ were wrong (too high) by a factor of more than fifty. Experimenters eagerly sought the phenomenon, but failed to find it at the predicted temperature: the theorists then increased the sophistry of their calculations and revised their estimates of $T_{\mathrm{c}}$ downwards, a cycle which was repeated several times. This procedure was so utterly demoralising for those doing the experiments (being the classical brainwashing technique of repeatedly raising the victim's hopes and then dashing them again) that, when the superfluid transition finally did manifest itself in Osheroff's cryostat at Cornell, it took several weeks for him and his coworkers to realise the significance of what they had discovered. Hence the notable reluctance, so far, of most theorists to predict a $T_{\mathrm{c}}$ for ${ }^{3} \mathrm{He}-{ }^{4} \mathrm{He}$ solutions.

A solution of a few percent ${ }^{3} \mathrm{He}$ in ${ }^{4} \mathrm{He}$ is stable down to the lowest temperatures and is, even without the expected transition, of considerable intrinsic interest. The ${ }^{3} \mathrm{He}$ atoms exist and move in a background aether-the liquid ${ }^{4} \mathrm{He}$ - which is itself a superfluid whose transition temperature lies at the relatively high value of $2 \mathrm{~K}$. At low temperatures, where the ${ }^{4} \mathrm{He}$ entropy is essentially zero, the ${ }^{3} \mathrm{He}$ atoms dominate the behaviour of the liquid. In fact, if one completely ignores the presence of the ${ }^{4} \mathrm{He}$ (except insofar as it results in each ${ }^{3} \mathrm{He}$ atom having an effective mass which is larger than the bare atomic mass), and simply treats the ${ }^{3} \mathrm{He}$ atoms as though they were an ideal gas, one gets the right answers for most of the liquid's properties.

Now, it is known that an attractive interaction occurs between the ${ }^{3} \mathrm{He}$ atoms of a solution and, at a sufficiently low temperature, this is expected to give rise to the formation of Cooper pairs, and hence to superfluidity. It is not yet clear whether the relative angular momentum of the atoms in a pair will be zero, as for the Cooper pairs of electrons in a superconductor, or unity as is apparently the case in superfluid pure ${ }^{3} \mathrm{He}$. The situation would certainly be very peculiar in either case, however, since the superfluid ${ }^{3} \mathrm{He}$ which was formed would exist in another, completely separate but interpenetrating superfluid, the ${ }^{4} \mathrm{He}$, which is fundamentally different in nature in that it does not acquire its superfluidity through the formation of Cooper pairs. The bizarre physical properties to be expected of such a system were discussed in considerable detail by Khalatnikov in his invited address (Khalatnikov, Mineev and Volovick: 